\title{
Strength and Skin Temperature Assessment: Comparing Active and Geriatric Populations
}

\author{
Carolina Magalhaes, Pedro Contente, Ricardo Vardasca, Paulo Abreu, Joaquim Mendes, Maria \\ Teresa Restivo
}

\begin{abstract}
The age-related consequence of loss of skeletal muscle strength can be evaluated by handgrip force (HGF) tests. The assessment of this parameter is performed with dynamometers and it is frequently used as a functional indicator of different pathologies. During gripping exercises, physiological alterations occur that can be quantified with infrared thermography (IRT), adding information to an individual's health status assessment. This work focus on the use of HGF and IRT measurements to evaluate differences among populations of active and institutionalized individuals, and on the identification of correlations between thermal parameters and HGF measurements. The study's population included 30 active adults and 32 institutionalized individuals. Each performed an established handgrip exercise, mechanically stimulating forearm muscles. IRT images of this body region were recorded during the entire experiment. Three regions of interest (ROIs) were established for thermal image analysis, encompassing the digital flexor muscle region and the wrist ulnar and radial artery zone. Differences between populations were verified for the collected data, showing higher HGF measurements (Maximum force, Average force and Accumulated handgrip work) and elevated skin average temperatures for active adults, when compared to institutionalized participants. Strong correlations between HGF measurements and thermal parameters were also encountered, suggesting its relevance for future research. Thus, the results demonstrate the importance of pairing different types of technologies to increase the range of information and confidence in the results for possible medical applications. Some usages may include the diagnosis, prediction and treatment monitoring of musculoskeletal pathologies, as rheumatoid arthritis, tendinitis and carpal tunnel syndrome.
\end{abstract}

Index Terms - active, geriatric, handgrip force, skin temperature.

\section{INTRODUCTION}

Degenerative anatomo-physiological changes are often associated with the aging process [1]. Muscle mass depletion is one of the consequences of this natural pathway that reflects itself through the reduction of muscle strength, particularly on upper limbs extremities, making handgrip force (HGF) an indicator of functional status [2], [3]. Apart from muscle impairment assessment, other studies have employed this

Carolina Magalhaes, INEGI-LAETA, Faculty of Engineering, University of Porto, Porto, Portugal

Pedro Contente, INEGI-LAETA, Faculty of Engineering, University of Porto, Porto, Portugal

Ricardo Vardasca, INEGI-LAETA, Faculty of Engineering, University of Porto, Porto, Portugal, +351 220414741

Paulo Abreu, INEGI-LAETA, Faculty of Engineering, University of Porto, Porto, Portugal

Joaquim Mendes, INEGI-LAETA, Faculty of Engineering, University of Porto, Porto, Portugal

Maria Teresa Restivo, INEGI-LAETA, Faculty of Engineering, University of Porto, Porto, Portugal parameter to verify the relation of HGF and oral functions, atherosclerosis, socioeconomic status and, more thoroughly, undernourishment, always on elderly populations [4]-[8] HGF has also shown usefulness for assessing muscle function and frailty [9], [10].

The assessment of HGF with hand-held dynamometers makes it a portable and low cost solution for straightforward clinical applications, without the needed of a trained specialist [11]. Still, there is not a consensus on the validity of HGF as an indicator of health status, due to its supposed dependency on several other parameters, as age, sex, Body Mass Index (BMI) and even individual activity levels [12]-[15].

There are few studies involving HGF measurements with groups of young and geriatric adults. Most of the found are centered on the use of gripping exercises as a stress stimulus to assess other pathology-related parameters [16], [17]. Samuel J. et al. collected cardiovascular data during the performance of gripping exercises to verify if isometric handgrip echocardiography (IHE) could be used to detect diastolic dysfunction. The studied population included 19 young healthy adults ( $24 \pm 4$ years) and 17 elder participants (72 \pm 6 years) with age-related abnormal diastolic function. The performance of HGF tests lead to a great variation of echo-cardial measurements in 11 elderly individuals, showing the usefulness of IHE for diastolic function assessment [16]. On a similar approach, by Hartog R. et al., implemented handgrip tests to evaluate variations on vascular hemodynamic responses. A total of 62 participants were divided in 3 age groups: 20-40, 41-60 and 61-80 years old, with 22, 20 and 20 participants respectively. After gripping tests, vascular hemodynamics were assessed, based on variations of pulsatile pressure of the brachial artery. The mechanical stress lead to increased blood pressure in elder individuals, correlating the HGF with vascular stiffness in older populations [17]. The implementation of HGF for non-diagnostic approaches involving young and senior individuals is even scarcer. With the goal of identifying force decline during continuous maximal handgrip for elderly people, De Dobbeleer L. et al. studied one experimental group composed with 91 hospitalized senior participants (83 \pm 5 years) and two controls involving elderly independent individuals (74 \pm 5 years) and young healthy adults $(23 \pm 3$ years), each with 100 volunteers. Different strength decays were encountered for different groups, relating HGF with age and health status [18].

Even though important data can be retrieved from HGF measurements, the addition of a different type of information could improve confidence on the conclusions achieved through the analyses of gripping test results.

Infrared thermography (IRT) is an imaging technique that allows non-contact and risk-free evaluation of skin surface 
temperature. The emitted thermal radiation is captured and represented in the form of thermograms, allowing an innocuous, inexpensive and fast physiological analysis upon a chemical, mechanical or thermal stimulus [19], [20]. Since aging handicaps not only muscular tissue, but also the vascular and neurological systems, IRT has the potential to detect real-time temperature variations during HGF tests that could indicate abnormal health conditions caused by musculoskeletal degeneration [21], [22].

This research aims to evaluate indicators of functional (HGF) and physiological (IRT) energy, and assess differences between active and institutionalized individuals, and to determine the relationship between thermal parameters and HGF measurements.

\section{MATERIALS AND METHODS}

The developed protocol uses the acquisition of IRT images during the execution of HGF exercises. To prevent unwanted motion of the forearm and ensure correct positioning throughout the entire test, a forearm support was manufactured and used during tests to maintain the upper limb still.

The images were collected using the thermal camera FLIR (Wilsonville, OR, USA) A325sc (Focal Plane Array sensor of $320 \times 240$, Noise-equivalent temperature difference of $<50$ $\mathrm{mK}$ at $30{ }^{\circ} \mathrm{C}$ and a measurement uncertainty of $\pm 2 \%$ of the overall reading). The equipment was connected to a laptop, allowing the visualization of thermal fluctuations during the test period and the immediate storage of images. To assure adequate room conditions, mean temperature $(\mathrm{T})$ and relative humidity (RH), were monitored $\left(\mathrm{T}=21.8 \pm 0.72^{\circ} \mathrm{C}\right.$ and $\mathrm{RH}=50.1 \pm 0.92 \%)$, in accordance with the international guidelines [20], [23]. For that, a hygrometer - Testo® (Lenzkirch, Germany) $175 \mathrm{H} 1$ (accuracy of $\pm 0.4^{\circ} \mathrm{C}$ and $\pm 2 \%$; resolution of $0.1{ }^{\circ} \mathrm{C}$ and $0.1 \%$ and an operational range of -20 to $+55{ }^{\circ} \mathrm{C}$ and 0 to $100 \%$ ) was used. Additionally, the occurrence of airstreams and incidence of illumination were avoided.

For the HGF measurements, a handheld prototype dynamometer was used (BodyGrip), which was developed at the authors' research group [24]. It eases the quantification of hand gripping forces through the use of wireless technology, enabling permanent communication with a computer.

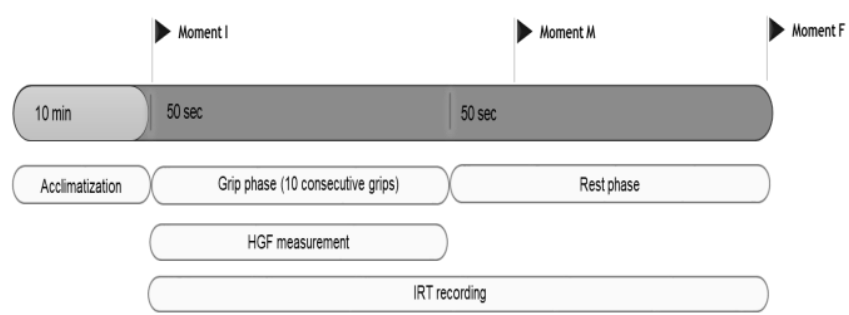

Fig. 1 - Scheme of the develop methodology for HGF measurement and IRT assessment.

The recorded grip forces are registered through an application installed in the PC. It allows the setting of test conditions, e. g., test duration, and it has the ability to estimate the energy, i.e., work, transmitted from the user to the device during a gripping test. Additionally, it is lightweight $(0.25 \mathrm{~kg})$ and presents a slim design $(0.144 \times 0.022 \times 0.045 \mathrm{~m})$ with a resolution of $1 \mathrm{~N}$ and a force range from 0 to $900 \mathrm{~N}$. For every measurement, the force $(\mathrm{N})$ and time $(\mathrm{ms})$ are calculated and storage in a file with CSV format. Thus, the calculated HGF measurements for each gripping exercise included: maximum force $\left(\mathrm{F}_{\text {Max }}\right)(\mathrm{N})$, average force $\left(\mathrm{F}_{\text {Aver }}\right)(\mathrm{N})$ and accumulated handgrip work $\left(\mathrm{W}_{\mathrm{Acc}}\right)(\mathrm{J})$. Maximum force corresponds to the maximum force measured for each grip, $F_{\text {Aver }}$ represents the average measured force during the entire gripping exercise and $\mathrm{W}_{\mathrm{Acc}}$ the sum of the mechanical work necessary for the achievement of maximum force at each grip.

Before conducting grip strength tests, each participant was subjected to a period of thermal acclimatization of 10 minutes. The test exercise started with the execution of 10 consecutive grips of 5 seconds each - grip phase. After the performance of the first grip with maximum force, the dynamometer was released, but not dropped, and another grip was performed immediately after, until the 10th-grip mark. A relaxation period followed, for 50 seconds, with the participant holding the device without the application of grip force - rest phase. This last phase was included to allow the recording of delayed temperature fluctuations in response to the application of a mechanical stimulus. During grip strength test, three different moments were established: $I$ - Before the beginning of grip phase; $M$ - time instant (at 15 seconds after the last grip) where all ROIs present a maximum temperature decrease; $F$ - End of resting phase. The choice of moment $M$ was based on previous work results [25]. Fig. 1 summarizes the protocol stages.

The relation between the collected IRT data and maximum HGF was then established based on the same time interval.

In total, 62 participants were included in this study. The sample was divided in 2 groups: an institutionalized group (26 women and 6 men) from the day care facility Centro Social do Bom Pastor (Vila Nova de Gaia, Portugal), and an active group ( 9 women and 21 men). Each participant received detailed information concerning the test and signed the written consent. Lastly, the age, sex and body mass index (BMI) were registered for each participant (Table I)

Table I - Average and standard deviation values of age and BMI, according to participants group.

\begin{tabular}{cccc}
\hline & Group & Age $(\mathbf{y})$ & BMI (\%) \\
\hline \multirow{3}{*}{ Active } & Global & $32.8 \pm 6.13$ & $25.9 \pm 1.67$ \\
& Female & $33.7 \pm 6.43$ & $24.6 \pm 1.62$ \\
& Male & $32.4 \pm 5.99$ & $26.4 \pm 1.62$ \\
\multirow{2}{*}{ Instituti } & Global & $76.4 \pm 4.88$ & $26.5 \pm 2.40$ \\
onalized & Female & $78.0 \pm 4.54$ & $27.5 \pm 2.39$ \\
& Male & $69.7 \pm 4.86$ & $22.5 \pm 0.97$ \\
\hline
\end{tabular}

For the analysis of the influence of gripping exercises in forearm temperature, three Regions of Interest (ROIs) where established (Fig. 2). The first ROI (ROI1) was set over the digital flexor muscle, since it is the main actuator during handgrip tests, ROI2 was placed over the wrist radial artery and ROI3 over the wrist ulnar artery, both vessels responsible for hand blood supply [26], [27].

The FLIR (Wilsonville, OR, USA) ThermaCAM Researcher Pro 2.10 software was used to process the recorded images and obtain, for each image, the average temperatures of each ROI, which were used to calculate the relative average temperature differences from baseline. Other thermal parameters were also calculated using average temperature values: difference between the average 
temperature at moments $M$ and $I(\Delta \mathrm{T} 1)$ and $\Delta \mathrm{T} 2$ between $F$ and $M$ for each ROI; gradient ( $\nabla \mathrm{T})$ of these differences $(\Delta \mathrm{T} 2$ $-\Delta \mathrm{T} 1$ ); temperature differences between ROIs (ROI1-ROI2, ROI2-ROI3 and ROI1-ROI3) at moments $I, M$ and $F$.

The average ROI temperature values and HGF measurements were analyzed through statistical methods using the IBM ${ }^{\circledR}$ (Armonk, NY, USA) SPSS v24 statistical analysis software package with a statistical significance lower than $5 \%$ for all tests. Variables normality was assessed with Kolmogorov-Smirnov test and in case of not all variables had statistical evidence of following the normal distribution, the non-parametric tests Mann-Whitney U and Kruskal-Wallis were used to assess the influence of participant group, age, sex and BMI on average ROI temperature values and HGF measurements. The same task was repeated using relative average temperature differences instead. Lastly, Spearman correlations were calculated between the other thermal parameters and HGF measurements (maximum force $\left(\mathrm{F}_{\mathrm{Max}}\right)$, average force $\left(\mathrm{F}_{\mathrm{Aver}}\right)$ and accumulated handgrip work $\left(\mathrm{W}_{\mathrm{Acc}}\right)$ ).

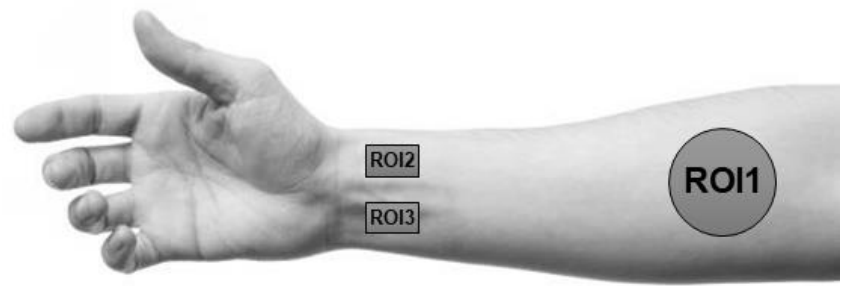

Fig. 2 - Selected regions of interest for thermal analysis of the forearm.

\section{RESULTS}

The maximum $\left(\mathrm{F}_{\max }\right)$ and average $\left(\mathrm{F}_{\mathrm{aver}}\right)$ handgrip force per participant group are presented in Fig. 3. In both measurements, active individuals surpassed by more than double geriatric participants.

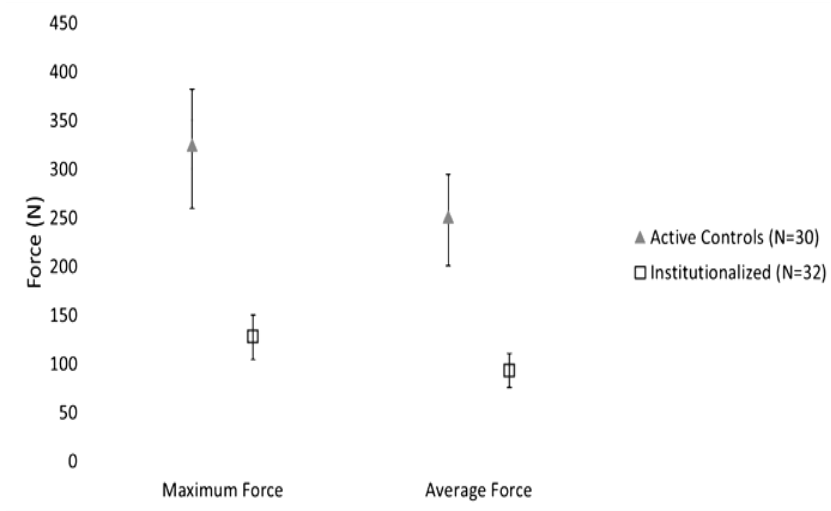

Fig. 3 - Maximum and average handgrip force according to group of participants.

The same tendency was verified in accumulated handgrip work with institutionalized participants performing substantially worse than active adults (Fig. 4).

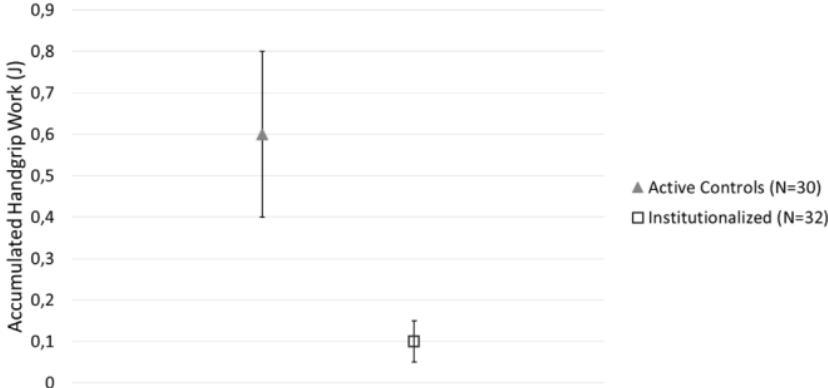

Fig. 4 - Accumulated hand grip work according to group of participants

Clear differences in the average mean temperatures of ROIs can be verified in Fig. 5, 6 and 7. Institutionalized participants demonstrated constantly lower temperatures than active controls, throughout the entire HGF test. This disparity is greater on the region of interest correspondent to the digital flexor muscle (Fig. 5) followed by wrist radial artery and wrist ulnar artery (Fig. 6 and 7, respectively).

The average temperature of ROI 1 of active controls was maintained around $33^{\circ} \mathrm{C}$, surpassing institutionalized individuals by $1.5{ }^{\circ} \mathrm{C}$ (Fig. 5). The active control group also displayed a slight increase in average temperature values towards the end of the gripping exercise.

The average temperature values encountered for ROI 2 were higher than those of ROI1, fluctuating around $33.3^{\circ} \mathrm{C}$ and $31.9^{\circ} \mathrm{C}$ for active controls and institutionalized participants (Fig. 6). As in ROI1, active controls showed a temperature increase during the rest phase.

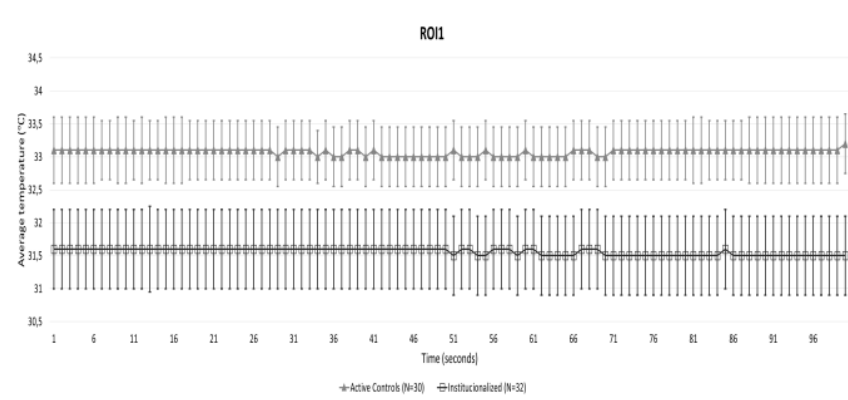

Fig. 5 - Average temperature of digital flexor muscle ROI for active and institutionalized participants during HGF test.

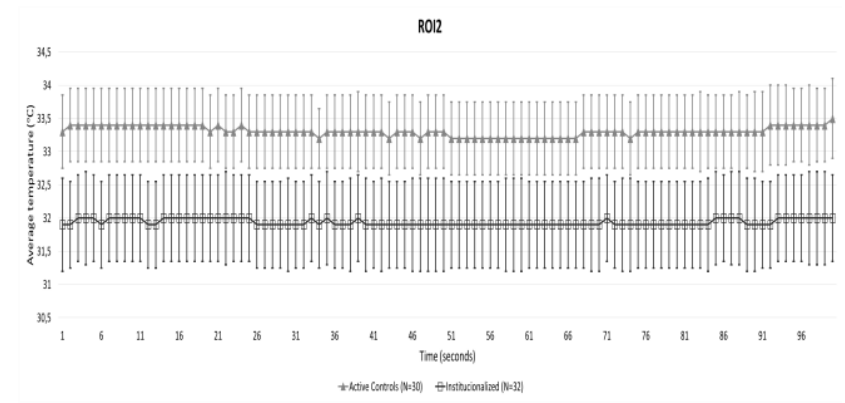

Fig. 6 - Average temperature of wrist radial artery ROI for active and institutionalized participants during HGF test. 
RO13

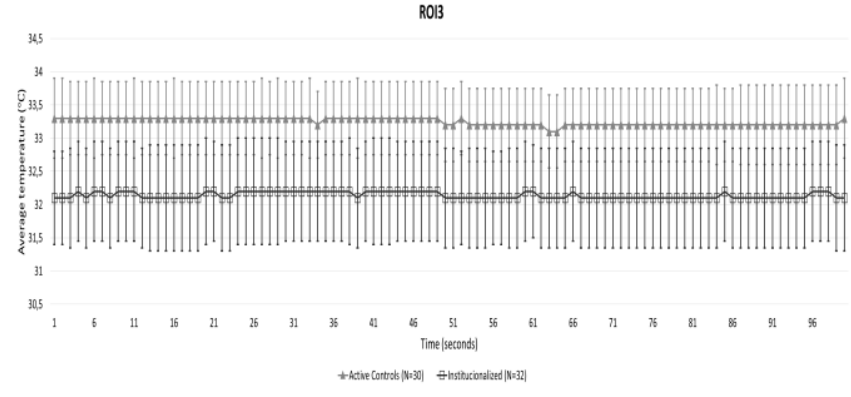

Fig. 7 - Average temperature of wrist ulnar artery ROI for active and institutionalized participants during HGF test.

Similarly to ROI2, on ROI3 both groups presented higher average temperature values when compared to ROI1, being, on average, $33.2^{\circ} \mathrm{C}$ and $32.1^{\circ} \mathrm{C}$ for active controls and institutionalized participants. On the final stage of resting, a rise on average temperature was verified, once again, on the active control group. Looking at average relative temperature differences, greater deviations from baseline were encountered for the active group during the performance of HGF tests, particularly on ROI2 (Fig. 9) and ROI3 (Fig. 10) respectively. While the institutionalized group showed a more randomized behavior throughout HGF tests, active adults displayed a decrease in temperature from the baseline towards the end of gripping exercises, returning to normality on the resting stage.

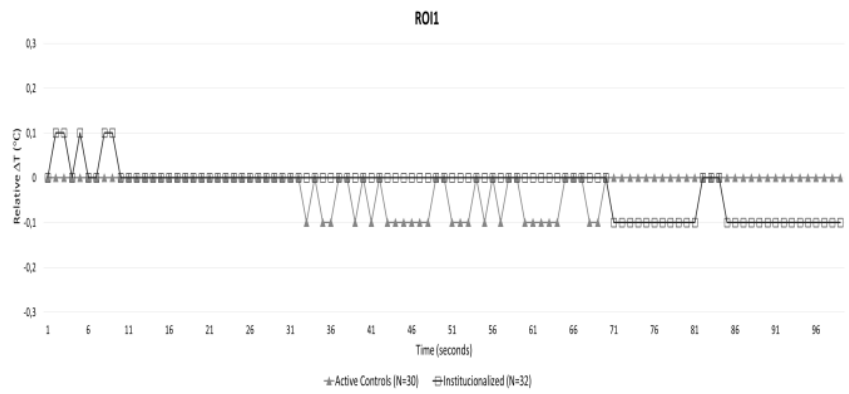

Fig. 8 - Average relative temperature differences from baseline of digital flexor muscle ROI for active and institutionalized participants during HGF test.

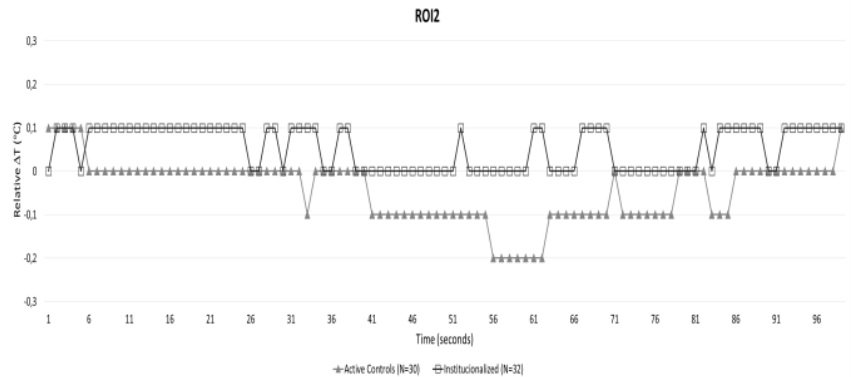

Fig. 9 - Average relative temperature differences from baseline of wrist radial artery ROI for active and institutionalized participants during HGF test.

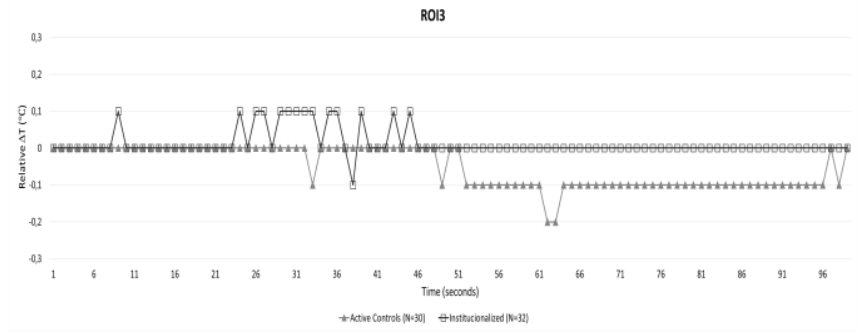

Fig. 10 - Average relative temperature differences from baseline of wrist ulnar artery ROI for active and institutionalized participants during HGF test.
The normality tests showed that not all the considered variables followed a normal distribution so the non-parametric tests Mann-Whitney $U$ and Kruskal-Wallis were used to verify the influence of participant group, sex, age and BMI in the collected values. Spearman correlation was calculated after to verify the correlation between other thermal parameters and HGF variables.

Statistical evidence of group type (Mann-Whitney U test, $\mathrm{p}<0.05)$ and age (Kruskal-Wallis test, $\mathrm{p}<0.05)$ was found in all average temperature values and HGF measurements. Sex (Mann-Whitney U test, $\mathrm{p}<0.05$ ) only influenced values collect from the wrist ulnar artery region (ROI3), while BMI affected none.

Considering relative average temperature difference, group type (Mann-Whitney U test, $\mathrm{p}<0.05)$ influenced ROI1 and ROI 2 at $I$ moment, ROI2 at $M$ moment and ROI1 at $F$ moment.

For other calculated thermal parameters, statistical evidence of group type discrimination (Mann-Whitney U test, $\mathrm{p}<0.05)$ was encountered in ROI2 $\Delta \mathrm{T} 1$, ROI $\Delta \mathrm{T} 2$, ROI2 $\Delta \mathrm{T} 2, \mathrm{ROI} 2 \nabla \mathrm{T}, \mathrm{ROI} 3 \nabla \mathrm{T}$ and ROI2-ROI3 at I moment. Age was found to influence (Kruskal-Wallis test, $\mathrm{p}<0.05$ ) ROI1 $\Delta \mathrm{T} 2$, ROI2 $\Delta \mathrm{T} 2, \mathrm{ROI} 2 \nabla \mathrm{T}$, and sex had an impact (Mann-Whitney U test, $\mathrm{p}<0.05$ ) on ROI3 $\Delta \mathrm{T} 1$, ROI3 $\Delta \mathrm{T} 2, \nabla \mathrm{T}$ of all ROIs, ROI1-ROI2 at $I, M$ and $F$ moments and ROI1-ROI3 at $I$ and $M$ moments. BMI presented statistical evidence (Mann-Whitney U test, $\mathrm{p}<0.05)$ on ROI2 $\Delta \mathrm{T} 2$ and ROI2 $\nabla \mathrm{T}$, ROI1-ROI2 I, ROI2-ROI3 I, ROI1-ROI3 I and $M$. Strong correlation (Spearman $\mathrm{p}<0.01$ ) was found between all HGF measurements and ROI1 DT2 and ROI2 $\nabla$ T. A weaker influence (Spearman p <0.05) was encountered on ROI2 $\Delta \mathrm{T} 1$, ROI2 $\Delta \mathrm{T} 2$ and ROI1-ROI3 at $M$ moment. Maximum force was also weakly connected to ROI1 $\nabla \mathrm{T}$, ROI3 $\nabla \mathrm{T}$ and Average force and Accumulated handgrip work to ROI3 $\nabla \mathrm{T}$, R1-R2 at $M$ and $F$ moments and ROI1-ROI3 at $I$ moment.

Table II summarizes Spearman correlation coefficients ( $\rho)$.

\begin{tabular}{ccccc}
\hline \multirow{2}{*}{$\begin{array}{c}\text { Spearman } \\
\text { correlation }\end{array}$} & $\begin{array}{c}\text { Other thermal } \\
\text { parameters }\end{array}$ & \multicolumn{3}{c}{ HGF measurements } \\
\cline { 3 - 5 } & ROI1 $\Delta \mathrm{T} 2$ & 0.565 & 0.544 & 0.543 \\
\hline $\begin{array}{c}\text { Strong } \\
\text { correlation } \\
(\rho<0.01)\end{array}$ & ROI2 $\nabla \mathrm{T}$ & 0.368 & 0.344 & 0.355 \\
\hline & ROI2 $\Delta \mathrm{T} 1$ & -0.28 & -0.258 & -0.26 \\
& ROI2 $\Delta \mathrm{T} 2$ & 0.291 & 0.292 & 0.313 \\
& ROI1 $\nabla \mathrm{T}$ & 0.292 & 0.263 & - \\
& ROI3 $\nabla \mathrm{T}$ & 0.289 & - & 0.268 \\
Weak & ROI1-ROI2 M & - & 0.288 & 0.290 \\
$\begin{array}{c}\text { correlation } \\
(\rho<0.05)\end{array}$ & ROI1-ROI2 F & - & 0.289 & 0.288 \\
& ROI1-ROI3 I & - & 0.273 & 0.272 \\
& ROI1-ROI3 M & 0.251 & 0.301 & 0.317 \\
\hline
\end{tabular}

The differences of temperature between moments on institutionalized participants showed that the ROIs average temperature remained the same for all forearm regions, except for a decrease between the moment $M$ and the moment $F$ on 
ROI1 (Fig. 11). For active controls, a decrease in temperature was registered on all ROIs between the first two moments ( $I$ and $M$ ). Contrarily, a rise occurred from moment $M$ to $F$. Both temperature fluctuations were more accentuated on the wrist radial artery region. Identically, in the analysis of gradients, ROI2 presented the greatest positive temperature difference in the active group (Fig. 12), while the institutionalized participants displayed negative variation on ROI1 and ROI3.

Focusing in temperature differences between regions of interest (Fig. 13, 14 and 15), the institutionalized group displayed similar values for all moments, with small differences between wrist ROIs, medium differences between ROI1 and ROI2 and greater differences in ROI1-ROI3. For every moment ROI3 presented a higher average temperature than ROI2 and ROI2 a greater value than ROI1. In active controls, ROI1-ROI2 and ROI1-ROI3 were equal in the moments $I$ and $M$, respectively (Fig. 13 and 14), while ROI1-ROI2 exceeded ROI1-ROI3 during resting phase (Fig. 15). Contrarily to geriatric participants, the wrist radial artery ROI displayed higher average temperatures than the wrist ulnar artery ROI during most moments (Fig. 13 and 15).

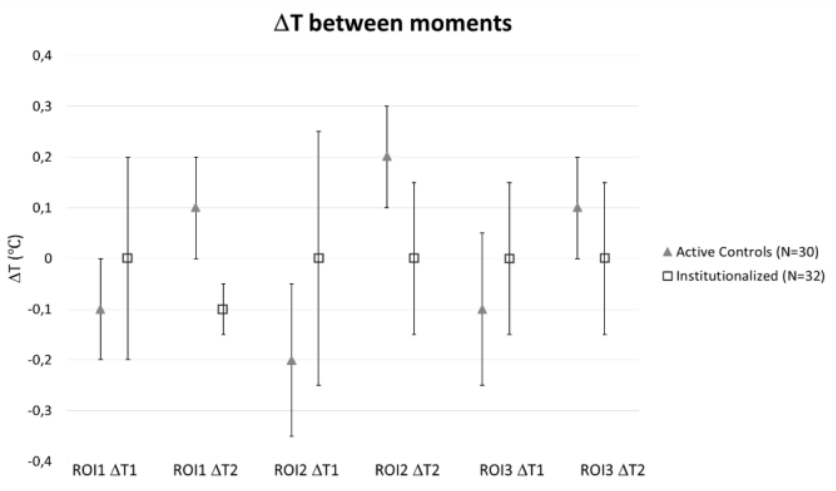

Fig. 11 - Differences of temperatures among moments I and M $(\Delta \mathrm{T} 1), \mathrm{M}$ and $\mathrm{F}(\Delta \mathrm{T} 2)$, for ROI1, ROI2 and ROI3 ( ${ }^{*} \mathrm{p}<0.05$; $* * \mathrm{p}<0.01)$.

$\nabla \mathrm{T}$

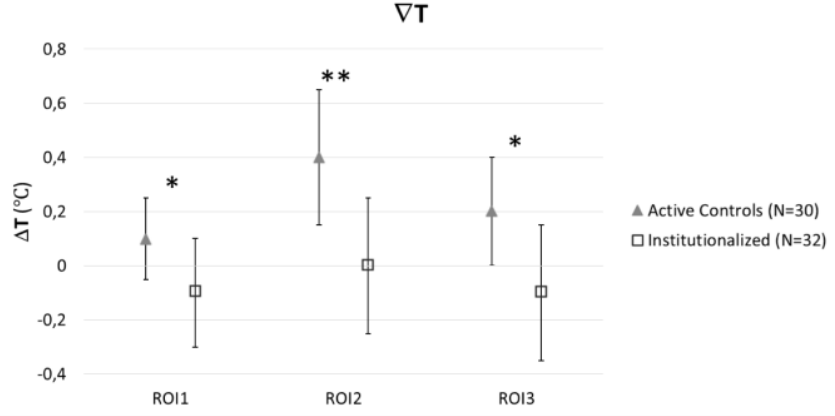

Fig. 12 - Gradients for ROI1, ROI2 and ROI3 (*p<0.05; **p<0.01).

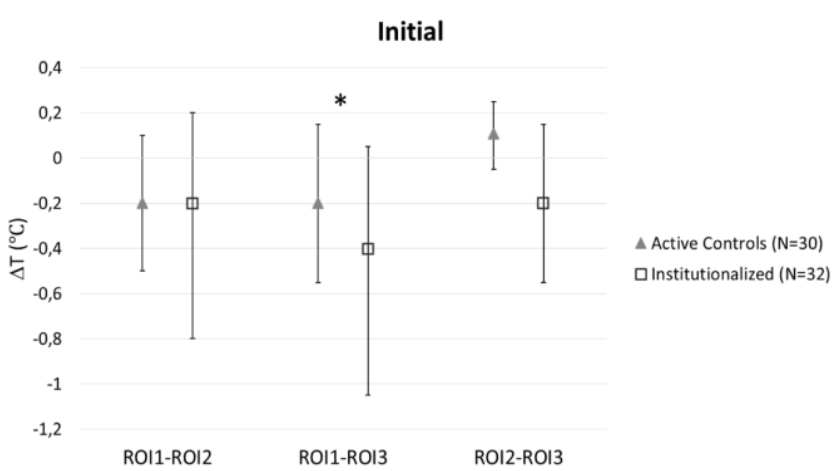

Fig. 13 - Temperature differences between ROIs at moment I $(* \mathrm{p}<0.05)$.

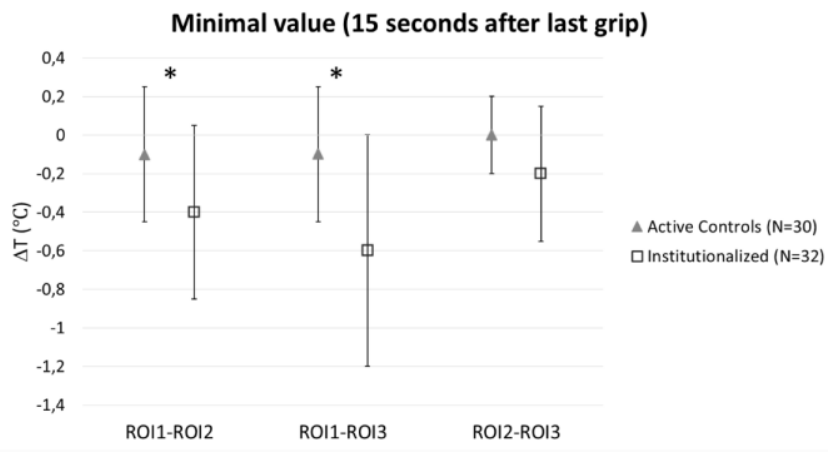

Fig. 14 - Temperature differences between ROIs at moment M $(* \mathrm{p}<0.05)$.

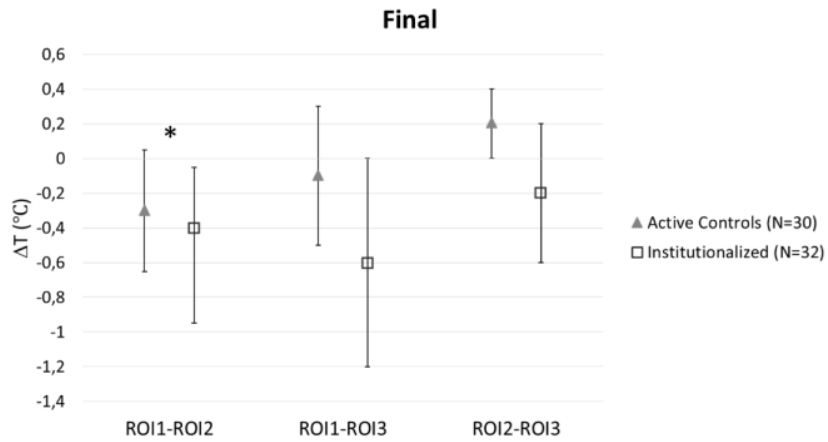

Fig. 15 - Temperature differences between ROIs at moment $\mathrm{F}$ $(* \mathrm{*}<0.05)$.

\section{DISCUSSION}

The present work involved the acquisition of forearm IRT images during the performance of HGF exercises. The studied population included 30 active controls and 32 institutionalized individuals. To the best of the authors' knowledge, this is the first study comparing thermal parameters and handgrip measurements between these two groups' types.

The surpass of institutionalized HGF measurements by active controls goes in accordance to what is commonly reported in the literature (Fig. 3 and 4) [2], [28]-[30]. Muscle mass depletion tends to aggravate with the aging process, so weakening of hand strength is verified in aging populations.

The impairment of vascular structures is also an associated consequence that affects peripheral circulation on more advanced ages, decreasing upper and lower limbs extremities temperature, as it has been described [31], [32]. This fact corroborates the inferior average temperature values encountered for institutionalized participants, during the performance of $\mathrm{HGF}$ exercises, when compared to active adults (Fig. 5, 6 and 7). The inability to properly control heat dissipation on upper extremities by elder individuals was also verified on the results of average relative temperature differences, since randomized small deviations from baseline were attested on institutionalized participants (Fig. 8, 9 and 10).

The encountered statistical influence of age on ROI2 $\Delta \mathrm{T} 2$ and sex on $\Delta \mathrm{T} 2$ variables has already been stated on other research [25]. However, the reported Spearman correlations between HGF measurements and thermal parameters differed from the ones verified in this study.

Nonetheless, correlations were found between thermal parameters and HGF measurements, particularly with ROI1 
$\Delta \mathrm{T} 2$ and ROI2 $\nabla \mathrm{T}$, suggesting its relevance for future research. It is worth mentioning that the accumulated work can be only estimated by some special dynamometers, through the measurement displacement feature, as the one used in this experiment.

The decrease of ROIs' average temperature during the first two moments ( $\Delta \mathrm{T} 1)$ followed by its increase from $M$ to $F$ $(\Delta \mathrm{T} 2)$ (Fig. 11) is consistent to what has been previously reported, concerning body temperature shifts during exercise [33]. This specific event was also shown in previous evaluations of active adults' forearms and could be a key factor for the distinction of healthy and muscle impaired subjects [25]. In addition, relevant information could be retrieved from wrist areas, due to the accentuated differences, among populations, of other thermal parameters (Fig. 12, 13, 14 and 15), and used for classification purposes.

The presented results show the relevance of combining different types of information (HGF and IRT) for medical applications. Some usages may include the diagnosis, prediction and treatment monitoring of musculoskeletal pathologies, as rheumatoid arthritis, tendinitis and carpal tunnel syndrome.

\section{CONCLUSION}

Clear differences were encountered between active and day-care institutionalized populations using HGF measurements and thermal parameters. The correlation between handgrip and thermal parameters was also identified. Apart from weaker correlations involving temperature differences between wrist zones and the digital flexor muscle area at different moments, it was found that ROI $\Delta \mathrm{T} 2$ and ROI2 $\nabla \mathrm{T}$ are strongly related to HGF measurements and its relevance should be explored in future studies.

The HGF technology by itself is cheaper than the IRT imaging and is able to discriminate alone the studied populations, but for a better quantification of all energies involved in the HGF exercise, the two technologies are required, which can provide better diagnostic data.

For upcoming research, it is suggested the combined implementation of $\mathrm{HGF}$ and IRT variables with machine learning classifiers to assess the usefulness of these parameters on population distinction, improving the outcomes of the assessments.

\section{ACKNOWLEDGMENT}

Authors gratefully acknowledge the funding of Project NORTE-01-0145-FEDER-000022 - SciTech - Science and Technology for Competitive and Sustainable Industries, cofinanced by Programa Operacional Regional do Norte (NORTE2020), through Fundo Europeu de Desenvolvimento Regional (FEDER). This work was also funded by Project LAETA - UID/EMS/50022/2013.

They also thank Prof. Angela Fernandes (ESTSP-IPP) for the establishment of contact with the day care facility Centro Social do Bom Pastor (Vila Nova de Gaia, Portugal), its director Dr. Sonia Vasconcelos and all the participants in the study for their precious time and collaboration.

\section{REFERENCES}

[1] I. Stambler, "Recognizing Degenerative Aging as a treatable medical condition: methodology and policy" Aging Dis. 2017, 8(5), pp. 583-589.

[2] F. Lauretani, et al., "Age-associated changes in skeletal muscles and their effect on mobility: an operational diagnosis of sarcopenia" J Appl Physiol. 2003 November, 95(5), pp. 1851-1860.

[3] B. H. Goodpaster, et al., "The loss of skeletal muscle strength, mass, and quality in older adults: the health, aging and body composition study" Journals Gerontol Ser A Biol Sci Med Sci. 2006, 61(10), pp. 1059-1064.

[4] A. M. Benjumea, C. L. Curcio, G. Duque, F. Gomez, "Dynapenia and sarcopenia as a risk factor for disability in a falls and fractures clinic in older persons" Open Access Maced J Med Sci. 2018, 6(2), pp. 344-349.

[5] Y. Mihara et al. "Association of handgrip strength with various oral functions in 82- to 84-year-old community-dwelling Japanese" Gerodontology. 2018, 35(3), pp. 214-220.

[6] H. Yamanashi et al. "Association between atherosclerosis and handgrip strength in non-hypertensive populations in India and Japan" Geriatr Gerontol Int. 2018, 18(7), pp. 1071-1078.

[7] B. Cheval et al., "Association of early- and adult-life socioeconomic circumstances with muscle strength in older age" Age Ageing. 2018, 47(3), pp. 398-407.

[8] K. Norman, N. Stobäus, M. C. Gonzalez, J. D. Schulzke, M. Pirlich, "Hand grip strength: outcome predictor and marker of nutritional status" Clin Nutr. 2011, 30(2), pp. 135-142.

[9] I. Bautmans, E. Gorus, R. Njemini, T. Mets, "Handgrip performance in relation to self-perceived fatigue, physical functioning and circulating IL-6 in elderly persons without inflammation" BMC geriatrics 2007, 7(1), pp. 5.

[10] P. Becker et al., "Consensus statement of the Academy of Nutrition and Dietetics/American Society for Parenteral and Enteral Nutrition: indicators recommended for the identification and documentation of pediatric malnutrition (undernutrition)" Nutr Clin Pract. 2015, 30(1), pp. 147-161.

[11] R . Alberto, F. Draicchio, T. Varrecchia, A. Silvetti, S. Iavicoli, "Wearable monitoring devices for biomechanical risk assessment at work: current status and future challenges-a systematic review" Int $J$ Environ Res Public Health, 2018, 15(9).

[12] R. S. Guerra, I. Fonseca, F. Pichel, M. T. Restivo, T. F. Amaral, "Handgrip strength and associated factors in hospitalized patients" $J$ Parenter Enter Nutr. 2015, 39(3), pp. 322-330.

[13] N. M. Massy-Westropp, T. K. Gill, A. W. Taylor, R. W. Bohannon, C. L. Hill, "Hand grip strength: age and gender stratified normative data in a population-based study" BMC Res Notes. 2011, 4(1), pp. 127.

[14] S. Hidese et al. (2018, April 25), "Relationship of handgrip strength and body mass index with cognitive function in patients with schizophrenia" Front Psychiatry [Internet]. vol:9. Available from: http://journal.frontiersin.org/article/10.3389/fpsyt.2018.00156/full

[15] R. A. Mattioli, A. S. Cavalli, J. A. B. Ribeiro, M. C. da Silva, "Association between handgrip strength and physical activity in hypertensive elderly individuals" Rev Bras Geriatr e Gerontol. 2015, 18(4), pp. 881-891.

[16] T. Jake Samuel et al., "Isometric handgrip echocardiography: A noninvasive stress test to assess left ventricular diastolic function" Clin Cardiol. 2017, 40(12), pp: 1247-1255.

[17] R. Hartog, D. Bolignano, E. Sijbrands, G. Pucci, F. Mattace-Raso, "Short-term vascular hemodynamic responses to isometric exercise in young adults and in the elderly" Clin Interv Aging. 2018, 13, pp. 509-514.

[18] L. De Dobbeleer et al., "Force-time characteristics during sustained maximal handgrip effort according to age and clinical condition" Exp Gerontol. 2017, 98, pp. 192-8

[19] M. Kaczmarek, A. Nowakowski, "Active IR-thermal imaging in medicine" J Nondestruct Eval. 2016, 35(19).

[20] E. Ring, K. Ammer, "The technique of infrared imaging in medicine" in Infrared Imaging, F. Ring, A. Jung, J. Zuber, Ed. London: IOP Publishing; 2015, pp. 1-10.

[21] JJ. Manson, D. Isenberg, S. Chambers, M. E. Shipley, J. T. Merrill, "Musculoskeletal examination" in Rapid Review of Rheumatology and Musculoskeletal Disorders, New York: CRC Press; 2014, p. 1-12.

[22] J. E. Gold, M. Cherniack, A. Hanlon, J. T. Dennerlein, J. Dropkin, "Skin temperature in the dorsal hand of office workers and severity of upper extremity musculoskeletal disorders", Int Arch Occup Environ Health. 2009, 82(10), pp. 1281-1292. 
[23] K. Ammer, "The Glamorgan Protocol for recording and evaluation of thermal images of the human body", Thermol Int. 2008, 18(4), pp. $125-129$.

[24] M. R. Quintas, M. T. Restivo, B. Santos, C. Moreira da Silva, T. F. Andrade. Dispositivo para medir força e energia musculares. Patent No. P300.3 - PP2; 2015.

[25] R. Vardasca, P. Abreu, J. Mendes, M. T. Restivo, "Handgrip evaluation: endurance and handedness dominance" in Smart Industry \& Smart Education, M. Auer, R. Langmann, Ed. Springer; 2019. pp. 507-16.

[26] F. H. Netter, "Section 6: Upper limb - elbow and forearm" in Atlas of Human Anatomy, 5th ed. Saunders Elsevier, 2010, pp. 424-39.

[27] F. H. Netter, "Section 6: Upper limb - nervovasculature" in Atlas of Human Anatomy, 5th ed. Saunders Elsevier, 2010, pp. 460-7.

[28] T. Abe, R. S. Thiebaud, J. P. Loenneke, "Age-related change in handgrip strength in men and women: is muscle quality a contributing factor?" Age (Dordr). 2016, 38(1), pp. 28.

[29] L. C. Vianna, R. B. Oliveira, C. G. S. Araújo, "Age-related decline in handgrip strength differs according to gender" J Strength Cond Res. 2007, 21(4), pp. 1310.

[30] D. Samuel, K. Wilson, H. J. Martin, R. Allen, A. A. Sayer, M. Stokes, "Age-associated changes in hand grip and quadriceps muscle strength ratios in healthy adults" Aging Clin Exp Res. 2012, 24(3), pp. $245-250$.

[31] L. A. Holowatz, C. Thompson-Torgerson, W. L. Kenney, "Aging and the control of human skin blood flow", Front Biosci. 2010, 15, pp. $718-739$.

[32] J. Mercer, "A comparison of thermal responses in hands and feet of young and elderly subjects in response to local cooling as determined by infared imaging", Thermol Int. 2004, 14(2), pp. 71-76.

[33] M. Gleeson, "Temperature regulation during exercise", Int J Sports Med. 1998, 19(2), pp. 96-99.

Carolina Magalhaes is a researcher at INEGI-LAETA (Portugal), graduated with a BSc in Bioengineering from Universidade Catolica Portuguesa and a MSc in Biomedical Engineering from the Faculdade de Engenharia da Universidade do Porto (Portugal). Her interests are in the field of Biomedical Engineering, Computer Vision, Machine Learning and Data Analysis.

Pedro Silva is a graduate from BSc in Biomedical Engineering at the Instituto Politecnico do Porto and a MSc in Biomedical Engineering from the Faculdade de Engenharia da Universidade do Porto (Portugal).

Ricardo Vardasca holds a BSc (hons) in Information Technology and a $\mathrm{PhD}$ in Medical Informatics from the University of South Wales (UK) and a degree in Computer Science Engineering from the Instituto Politecnico de Leiria (Portugal). Actually, he is an integrated researcher at INEGI-LAETA (Portugal), research fellow at the Faculdade de Engenharia da Universidade do Porto (Portugal), external professor at the Faculty of Medicine and Odontology of University of Valencia (Spain), visiting fellow at University of South Wales (UK) and visiting researcher at University of Wollongong (Australia). He acts as general secretary of the European Association of Thermology and is a Fellow of the Royal Photographic Society.

Paulo Abreu is an assistant professor at the Mechanical Engineering department at the Faculdade de Engenharia da Universidade do Porto (Portugal) and an integrated researcher at INEGI-LAETA (Portugal). He holds a degree in Mechanical Engineering from the Faculdade de Engenharia da Universidade do Porto (Portugal), Msc in Industrial Robotics from the Cranfield Institute of Technology (UK) and a PhD in Mechanical Engineering from the University of Bristol (UK). His interests are in the field of robotics, actuators and mechatronic systems, both for industrial use and for rehabilitation.

Joaquim Gabriel has a Mechanical Engineering degree, a post-graduation degree in Automation and Management of Industrial Processes and a MSc in Industrial Computing from from the Faculdade de Engenharia da Universidade do Porto (Portugal), and a PhD in Industrial Electronics, Piezoelectric based applications, from Universidade do Minho (Portugal). From 1995 to 97 was EU-STA researcher at Kanagawa Science Park, Japan, working in very high precise positioning devices. Since 2003 he is Assistant Professor at the Mechanical Engineering department at the Faculdade de Engenharia da Universidade do Porto (Portugal), teaching automation and instrumentation subjects, and an integrated researcher at INEGI-LAETA (Portugal). In 2012 he was invited professor at Yokohama City University, Japan. He holds several patents in area of instrumentation. $\mathrm{He}$ has been developing instrumentation for applications, both in engineering and medicine. He is a member of the European Association of Thermology.
Maria Teresa Restivo has a Physics degree in Solid State Physics and a Ph.D. in Engineering Sciences, both at the University of Porto (Portugal). Her teaching activities are within the Automation, Instrumentation and Control Group of the Mechanical Engineering Department, Faculdade de Engenharia da Universidade do Porto (Portugal). Her research activities are within the System Integration and Process Automation Research Unit (UISPA) in the Associated Laboratory for Energy, Transports and Aeronautics (LAETA) funded by the Portuguese Science and Technology Foundation and hosted in the Research Pillar of the Institute of Science and Innovation in Mechanical and Industrial Engineering (INEGI). She was Member of the Faculty of Engineering Scientific Board since 2001-19. She is the head of the UISPA within INEGI-LAETA

Currently, topics of interest of applied research are among the development of Medical Instrumented Devices, Smart Devices and Online Experimentation and the Use of Emerging Technologies in Training and in Education.

She has participated in the creation of several non-formal learning activities for the Society organized at University of Porto and by its Faculty of Engineering and collaborated with some of those editions.

She is author (co-author) of articles and 6 books at National and International publishers (one awarded at national and international levels, among different other prizes in $\mathrm{R} \& \mathrm{D}$ ). She has been project co-ordinator and team member at national level and as FEUP's partner in European projects. She has been involved in supervising MSc and PhD theses. She has five national patents and one international. Two international claims are still pending. She is institutional member of the Global Online Laboratory Consortium (GOLC), Co-Chair of the Scientific Advisory Board of International Association of Online Engineering (IAOE). She is Past-President of International Society for Engineering Pedagogy (IGIP) and member of its Executive Committee. She was the first President of the Portuguese Society of Engineering Education (2010-12). She has the "ING PAED IGIP" diploma of International Engineering Educator. 